\title{
Hematology in the United Kingdom
}

By Archie Prentice, MB ChB, FRCP, FRCPath

This article is the first in a series of features comparing and contrasting aspects of oncology care delivery in non-US settings. The Journal of Oncology Practice will occasionally publish similar pieces in anticipation of discovering best practices from international health care systems. Dr Prentice's contribution is based on his presentation to the Committee on Practice of the American Society of Hematology at their December 2005 meeting.

\section{Structure and Function of the National Health Service}

A description of any medical specialty in the United Kingdom requires an explanation of the National Health Service (NHS). The NHS was created after the Second World War to equitably provide comprehensive and free medical care. Currently, most hospital-based medical specialists are salaried, full-time employees of the NHS, but a substantial minority are part-time and also practice privately. Nursing and all other staff in hospitals are full-time NHS employees, but private agencies supply some nursing and technical staff on part-time and short-term contracts. Roughly 250 hospitals cover local populations ranging from 100,000 to 600,000 people. Most function as District General Hospitals (DGHs), providing core medical and surgical services; some bigger hospitals provide more advanced care and function as tertiary referral, regional, or national specialty centers. These centers are usually in large cities and are attached to medical schools. Most hospitals do not have the recommended number of specialists for their population.

General practitioners (GPs) work as self-employed family doctors in community teams and employ their own staff. GPs are contracted to the NHS to provide primary care, with a complex system of remuneration known as "mixed block and itemized services." Referrals for specialized care are made by the patient's GP. Patients have an unlimited choice of hospitals, but in practice, most attend their local DGH. Patients cannot self-refer directly to specialist care unless they opt out of the NHS into the private sector. GPs have been encouraged to develop within their practices some of the lessintensive outpatient procedures that were traditionally confined to hospital specialists. There is no national shortage of GPs, but some economically disadvantaged areas have too few.

The UK government funds the NHS from national tax revenue. Within a 5-year financial planning cycle, the total annual budget for health care is declared, but full and accurate cost determination of individual medical procedures or packages of care is unknown. The government's Department of Health has tried in many different ways to distribute funding to target specific national problems (e.g., cancer and heart disease) and has set politically sensitive targets to improve performance (e.g., waiting times for elective care). The national need for health care has never been identified accurately; the total workforce and the skill mix needed to meet this demand are also unknown; and various funding formulas have never been sensitive enough to regional variations, in terms of either demographics or need.

The NHS is managed by layers of local and regional managers who are accountable to the Department of Health. They seem to be in an almost permanent state of reorganization. The numbers of local and regional managers have increased greatly in recent years as the government has attempted to command and control performance centrally. Many hospitals have now accumulated funding shortfalls of between $£ 10$ million and $£ 30$ million (\$18.6 million to $\$ 55.7$ million US). In 20042005 , the total NHS deficit was just under $£ 1$ billion ( $\$ 1.8$ billion US). In contrast to past years, the government has refused to find the extra cash to cover this shortfall, blaming it on local inefficiencies and financial mismanagement.

Hospitals are now trying to impose cuts in funding and staff while maintaining service. Note that although the hospital sector is in financial trouble, the GP sector is not.

\section{Provision of Clinical Hematology in the NHS}

On receipt of an abnormal blood test result, most patients are referred by a GP either to a hematology outpatient clinic or to the inpatient care of a consultant hematologist. Consultant hematologists are expected to make accurate diagnoses and treatment plans for all patients with malignant and nonmalignant hematologic disorders. However, increasing centralization has developed within regional or national networks of hospitals for specialized care. In some subspecialty areas, such as hemophilia care and pediatric hematology, centralization is well-organized and well-funded. Stem-cell transplantation has developed mainly in large teaching centers; however, there continue to exist many medium-sized hospitals and hematology units that perform fewer than 20 transplantations per year. In other subspecialty areas, such as general adult hematology-oncology, networking has been less well organized. For example, the current standard practice in the care of adult acute leukemia is remission induction in the DGH. This loose network of DGH consultants performed well in the Medical Research Council (MRC) randomized controlled leukemia trials, suggesting that this decentralized model may be effective in general adult hematology-oncology. 


\section{Attempts to Control Patient Access to Care Through NICE}

In the 1990s, the Department of Health created the National Institute for Clinical Excellence (NICE) to examine quality of care within the NHS. Outcomes of cancer care for solid tumors appeared to compare unfavorably with those of other countries in the European Union. Therefore, NICE began a series of cancer reviews (Improving Outcomes Guidance [IOG]) intended to produce uniform and better diagnosis and treatment. Evidence suggests that centralized care benefits patients with some cancers (e.g., breast, gastrointestinal, and gynecologic), so NHS care of these patients has been reorganized to allow access of larger numbers of patients to fewer specialist services.

The evidence of poorer outcomes in the care of most hematologic malignancies was less clear, though there seemed to be variable performance in lymphoma histopathologic diagnosis. Nevertheless, the IOG "one size fits all" approach has also been applied to the care of leukemias and lymphomas. Regional cancer networks are now trying to reorganize referral pathways to comply with the IOG's measures of quality of care for hematologic malignancy, centralizing more intensive aspects of the care of acute leukemia. It remains to be seen whether the new NHS can continue to deliver improved outcomes in the way that previous MRC trials demonstrated, while reducing duplication of services.

One shortcoming of NICE has been its disconnection from the evidence-based guidelines on the investigation and treatment of blood disorders, which were produced by members of the British Society of Hematology through its subcommittee, the British Committee for Standards in Hematology (available at www.bcshguidelines.com). These guidelines are intended to keep hematologists in the UK current with latest clinical developments, including the potential utility of new therapies.

NICE is also charged with making authoritative recommendations on the introduction of new therapies. The NICE recommendations and reorganization may have the effect of preventing local health managers from blocking patient access to drugs and therapies based on expense (known as "postal code prescribing"). Unfortunately, some local managers still block funding and access even when NICE recommends a new treatment, and even when a new drug is part of a nationally agreed-upon trial. For example, two recent high-profile cases were brought to the courts to contest denial of access to trastuzumab; in both cases, the judges ruled in favor of patient access to the drug.

\section{Provision of Hematology Laboratory Services in the NHS}

Much of the DGH hematologist's time is spent providing advice on the management of patients who are in the care of other colleagues, but have been discovered to have abnormal clinical laboratory results. This arises because, in practice, most hematologists provide a diagnostic laboratory service (analysis of peripheral blood and bone marrow, hematinic assays, coagulation studies, and oversight of blood transfusion) to other hospital specialists and to GPs. These providers depend heavily on this mix of laboratory and clinical consultative support in the treatment of their patients.

Until recently, hematology consultants have also had the additional role of hands-on leaders of teams of dedicated laboratory professionals; however, the growing demands of direct clinical care are pulling many away from the clinical laboratory. To fill the void, clinical scientists and technicians are showing an increasing interest in acquiring the work of physician medical graduates, benefiting from their leadership role. Recognizing the developing responsibility of the clinical scientist, the Royal College of Pathology is extending training and access to the MRCPath diploma in hematology to clinical scientists.

Highly specialized laboratory tests are centralized in reference laboratories, which operate within a centralized clinical practice in large teaching centers. The government is considering modernization of NHS pathology services, through centralization, rationalization, or even reduction in the services offered to patients. In large urban areas, centralization of laboratory services may mean that not all neighboring hospitals will be able to maintain a full range of clinical laboratory tests; combined (i.e., hematology plus biochemistry) laboratories will be more common. Modernization may also result in provision of services by private entities, with subsidy from NHS taxation revenue. It is possible that commercial for-profit clinical laboratories will displace NHS laboratories, which are cost-efficient, as a way of making a political point. Unfortunately, private care providers in the UK have shown little interest in delivering teaching and research within their service provision.

Despite these problems, NHS diagnostic hematology laboratories are excellent examples of increasingly costefficient organizations, which produce high-quality work that is very tightly controlled by a national external quality assurance scheme and a stringent national accreditation program according to ISO standards.

\section{Training Hematologists in the NHS}

The NHS has developed training to maintain and expand the numbers of consultant specialists, and those trainee numbers have steadily increased during the last 10 years to keep pace with the growth of the specialty. However, given the observed trends toward more part-time practice by both male and female physicians and impending early retirements, the number of training program graduates may still not be sufficient to satisfy the growing demand for specialty care. 
In the last 30 years, training has been structured to provide a generic hematology consultant who is able to handle most aspects of clinical practice as well as diagnostic clinical laboratory practice. Trainees who plan to enter higher specialist training in hematology are expected to have postgraduate general medical training and to have passed the membership examination of the Royal Colleges of Physicians (MRCP). Regional training programs lasting 6 years are attached to postgraduate deaneries and are approved by a national Joint Committee on Higher Medical Training. Completion of these programs usually coincides with passing the membership examination of the Royal College of Pathologists (MRCPath Haem). Advancing from medical school graduation to certification as a registered specialist in hematology takes about 8 years, or several years longer if a higher research degree (MD or $\mathrm{PhD}$ ) is undertaken. The government is now trying to enforce shorter postgraduate training times across all specialties. This may not affect the total time needed to train in hematology or the Royal College examination structure, but it will make taking time to earn an $\mathrm{MD}$ or $\mathrm{PhD}$ potentially more difficult.

The hematology specialty has been able to convince the Department of Health Workforce Review Team (WRT) that greater numbers of hematology training posts are needed each year. This effort has been assisted by the Royal College of Pathologists. Almost all of the new training posts made available are taken up, even when no new funding is made available centrally for them.

\section{Hematology Workforce Issues in the UK}

More than 700 consultant hematologists practice in the UK, all based in hospitals. Most DGHs have hematology departments. The distribution of hematology posts is uneven; large numbers are concentrated in big-city teaching hospitals, which allows subspecialty practice (e.g., in transplantation, bleeding disorders, and hemoglobinopathies). A few solo consultants remain, but only in the more remote and small hospitals. Currently, of the 700 government-allotted hematology posts, approximately 60 vacancies exist. Despite these vacancies, the expansion in consultant posts continues in both the local DGHs and the tertiary teaching hospitals. The WRT monitors the need for growth in consultant and trainee posts and has also now recognized the need for more consultant posts for a growing number of ethnic minority patients (particularly children with sickle cell disease) and for other subspecialty areas (e.g., pediatric hematology).
This expansion of consultant posts has been further fueled by the EU Working Time Directive, which determined that physicians (both consultants and trainees) should not be working more than 48 hours per week. Many think this determination, which has the force of law, is patently absurd because it fails to recognize the need to expose trainees to sufficient experience in emergency care. Nevertheless, the directive has been put into place by the government, which seemed surprised to find that NHS specialists had been working on average well over 60 hours per week. As a consequence, the government has been forced to either pay for the extra hours being worked in a new time-sensitive contract, or create new posts.

\section{Future of Hematology in the UK}

The structure of delivery of care and the role of the consultant hematologist may change significantly, but such a big and complex system of health care as the NHS could not function as effectively or efficiently without the hematology specialist. Concerns are growing that restricted training and funding (particularly in universities) are threatening the future of academic hematology, but that topic would need a separate review. However, the NHS WRT accepts the need for expanding the number of consultant hematologists to cope with the many and various demands made on the specialty, both in the clinic and in the laboratory. IOG reorganization is likely to increase the need for the specialist consultant hematologist in leukemia and lymphoma care, and more subspecialty hematologists than ever are needed to care for patients with nonmalignant disorders, such as inherited clotting disorders and hemoglobinopathies.

NHS managers and physicians often look abroad for examples of how to deliver a better service. It is difficult to find comparable systems and easy for us to be complacent about the unique and altruistic nature of the UK's service, in our belief that no other country has lessons for us. However, the NHS and hematology could surely benefit from some disinterested, critical, external appraisal.

Archie Prentice, MB, ChB, FRCP, FRCPath, is a consultant hematologist at The Royal Free Hospital and honorary senior lecturer at University College, both in London, England. He is the immediate past-president of the British Society for Haematology, chair of the British Committee for Standards in Haematology, co-chair of the CME Committee of the European Haematology Association, and chair of the Intercollegiate Committee for Haematology. 